\title{
Traffic-based floor preference for the scheduling of elevators in elevator group control system
}

\author{
Malan D. Sale, V. Chandra Prakash \\ Department of Computer Science \& Engineering, K.L.E.F., India
}

\begin{tabular}{l}
\hline Article Info \\
\hline Article history: \\
Received Jan 25, 2019 \\
Revised Apr 17, 2019 \\
Accepted May 10, 2019 \\
\hline Keywords: \\
Elevator Group Control \\
Systems (EGCSs) \\
Scheduling \\
Traffic patterns
\end{tabular}

Article Info

Aticle history:

Received Jan 25, 2019

Revised Apr 17, 2019

Accepted May 10, 2019

Keywords:

Systems (EGCSs)

Traffic patterns

\begin{abstract}
Modern high-rise buildings require complex yet efficient Elevator Group Control Systems (EGCSs). In vertical transportation through an elevator, a passenger must make a hall call by pressing a landing call button installed at each floor and located near the cars of the elevator group. Conventionally, the EGCS allocates one of the cars for each hall call. Waiting time for the arrival of car and journey time inside a car are two parameters, which provide a suitable measure for quality and efficiency of EGCSs. The proposed system deals with this car-call allocation problem. The proposed work analyzes the generated traffic patterns to dispatch a certain number of cars to certain floors in order to reduce the overall wait time of passengers. The proposed algorithm is simulated for high-rise building with 20 floors and provides a better result with the reduced wait time for more number of passengers.
\end{abstract}

Copyright (C) 2019 Institute of Advanced Engineering and Science. All rights reserved.

\section{Corresponding Author:}

Malan Dipak Sale,

Department of Computer science \& Engineering,

Vaddeswaram, Andhra Pradesh, India.

Email: mdsale2006@gmail.com

\section{INTRODUCTION}

With the fast development in technology, better utilization, and optimization of systems, EGCS has become a crucial area of concern. The Elevator framework has been a routine for almost all the buildings of modern society. For the clear majority, waiting for elevators until it arrives is the utmost upsetting role, but also the time it takes to reach the destination floor is not at ease too. This study emphasizes on condensing the waiting time of passengers. In the arena of elevator traffic, there exist various traffic forms, i.e., up-peak, down-peak, and inter-floor traffic patterns [1]. Up-peak traffic situations subsist when the prevailing stream is in an upward way when mainstream passengers are concerned about arriving the system from the main terminus of the building. In case of down peak traffic, the prevailing stream is in a downward way, when the majority of passengers are concerned about leaving the system from the main terminus of the building. Inter-floor traffic includes all the interchanges other than in up-peak and down-peak traffic.

Conventional elevator framework assimilates car call controls positioned near the elevator shaft. Passengers operate over these controls to make their respective calls for moving either in the upward or downward direction from their present floor. Once the EGCS allocates the elevator for a particular request, the passengers are supposed to push the destination floor on the displayed operating board of floors. Based on the above inputs, the elevator then dispatches towards the goal. Many dispatching algorithms have been presented for elevators as per the passenger needs. Few cases are designed in a way to handle specific traffic situations discussed above, while some work focuses on minimizing the total waiting time for passengers. For these kinds of structures, the elevator controller, handling the dispatching of elevators remains unaware of the goal floor of passenger until and unless the passenger explicitly selects one from the operating board. Because of this way of functioning, such structures are unable to recognize the number of passengers, waiting to be served or how many passengers are correlated behind a car call. For instance, a group of people might 
be interested in going to the same destination floor. However, the request for the elevator is sent only once, which happens mostly. In the account of elevator traffic, a lot of work has been done concentrating on various standpoints like minimizing waiting time of passengers, optimizing the model for specific traffic patterns, car-call allotment and much more. Literature shows that it is feasible to get the count of the passengers waiting to be served, by analyzing the historical traffic models or by mounting sensor devices near the elevator. However, these applications are useful only in specific suitable circumstances like deriving rules from historical patterns [2]. This may lead to the additional waiting time for passengers even if there is a slight change in traffic behavior. In the case of the mounting sensor, there involves an extra cost for the devices, and there might be some privacy concerns that can inhibit the device installation. Thus, there is a need for an optimal dispatching algorithm that can serve most of the requests with a minimal waiting time that can apply in general purpose buildings of day-to-day life. The study [3] presents a genetic algorithmbased approach for improving the waiting time of elevators. The genetic algorithm works by a natural development process. The algorithm took several floors, some cars, passive time, inter-floor trip time, hall call floors, car floors, car destination floors and based on this fitness calculation is done. The study focuses on random traffic instead of general traffic for waiting time optimization. The study [4] focuses on traffic identification by using k-means clustering method. The traveling salesperson problem is used to solve the elevator-dispatching problem where the elevator is visited every floor only once. The study [5] focuses on the use of Bayesian network to determine the event probability. The scheduling of elevators uses fuzzy rules. The count of passengers waiting at the floor and their waiting time is used to assign priorities to floors as high medium and low. The study [6] focuses on traffic pattern recognition using a fuzzy neural network. The identified patterns up-peak, down-peak and inter-peak traffics can use for further scheduling studies.

The study [7] presents an approach for modeling the power consumption of elevators and elevator dispatching according to elevator traffic. It works on reducing the waiting time of the elevator and minimizing the number of stops in order to lessen the journey time based on traffic. For each car call, the waiting time of passengers for each elevator is computed. Afterward, the elevator with the minimum projected waiting time is preferred and then this floor call request is added to the stopping list of elevators. For most of the proposed procedures in the literature, the routing characteristic is not contemplated. The study [8] compares multiple elevators dispatching approaches with a design, defining the elevator routes overtly. Both mean waiting time and mean journey time is considered as unbiased functions in the comparisons. In addition, they are validated with different traffic patterns for identification of the finest resolutions of these procedures. The research [9] proposes an even-odd elevator scheduling approach in EGCS. An even numbered elevator serves the even floors, and odd numbered elevators serve the odd floors. The fuzzy system approach is proposed in [10] for elevator scheduling in EGCS. The method is best suitable in a standard residential building. A methodology proposed in [11] appraise the probable checks of passengers for the origin-destination sets of each lift trip happening in a building. These tallies are utilized to make passengers traffic gauges which, thus, are utilized in lift dispatching to diminish vulnerabilities identified with future passengers and hence to move forward passenger's service level. The displayed methodology guarantees to beat previously suggested methodologies in terms of value and can take the favorable position of earlier data. For conserving energy, novel elevator scheduling approaches are presented in [12-13] that contemplate the dynamic variations of electricity price and passenger traffic. In a skyscraper construction, for transporting material goods and workers, an approach is presented in [14], which uses the zoning-based methodology.

The outline of a 3-dimensional elevator system is provided in [15] relying on a theory that elevator cars can move in all three paths. The regulatory controller dispatches elevator cars up a first vertical shaft to the destined floor. Finally, all passengers are delivered in scanner manner. The study [16] focuses on range assignment of elevator and floors for variable traffic. Four range assignment strategies are discussed; elevator visits to all floors, zoning based, specific floors in two range. The study considers the first floor as a starting and ending floor. The study focuses on floor assignments to elevators based on request cost, operating cost and lifting cost. Disjoint range assignment algorithm selects the elevator with minimum cost and overlapped range assignment algorithm selects an elevator to serve the range of floors and the highest request floor. The study [17] focuses on a new paradigm to find the benchmark value used to evaluate the efficiency of various elevator-dispatching algorithms. The study considers future knowledge of the passenger's destination is available.

The proposed scheduling approach reduces the number of elevator stops, as controller allocates explicit elevators to the identified high traffic floors. As compared to traditional elevator scheduling where each elevator stops at every floor, the even-odd scheduling gives less waiting time, as the number of elevator stops reduced. As compared to even-odd scheduling, the traffic-based floor preference scheduling gives the least waiting time. Numbers of elevator stops reduced as the controller do explicit elevator allocation to high traffic floors. 


\section{PROPOSED SYSTEM}

The functioning of the system broadly takes place in three stages; the gathering of passenger information, learning and recognizing the traffic pattern and dispatching of elevators. At the first stage, a mechanism is used to gather the traffic information, which includes the number of passengers going to a particular floor, source floor and the destination floor of the journey. Consistently, these coming traffic statistics are captured and conserved. In the second stage, this collected traffic information is used to comprehend traffic patterns out of this. Here the decision of optimal dispatching policy takes place based on the identified traffic pattern and thus further deriving high significance floor and the intensity of requests coming to those respective floors. At the final stage, this recognized traffic pattern knowledge is used in the arrival call allotment and the dispatching of elevators to the floors.

\subsection{Gathering Passenger Information}

To capture the passenger call information, a hypothetical building with 20 floors and four elevators with the hospital, shopping mall and an office at 10th, 15th, and 17th floor is considered. Here the Data generator generates some random data with required timestamps. These statistics saved in the database in the form of a file. As and when any latest information is available, the data is captured, and the same is imparted to the controller on the interval basis.

\subsection{Measurement of Passenger Traffic}

The numbers of passengers willing to enter the elevator are considered as a variable for calculation. To identify the passenger traffic movement in a building; the elevator capacity and the captured information are operated. The advantage with these indicators is that passengers need not worry about the measurement whatsoever. The elevator controller derives these patterns based on the rules engine defined by observing the general patterns of traffic flow in the building.

\subsection{Elevator Dispatching}

The controller considers identified traffic patterns during the allocation of elevators. As per the derived pattern, the highest priority floors are recognized. The dispatching policy will then allocate an explicit elevator to these floors in order to serve most of the requests coming from these significant floors with minimal waiting time for passengers. The controller considers the intensity of requests, to decide the number of elevators needs to allocate for the high priority floors. However, all elevators will serve to all floors, so, as the pattern changes these elevators become available to serve other floors. There will always be one or more elevator kept aside by the controller to serve calls coming from other floors than priority floors. The proposed system is as shown in Figure 1.

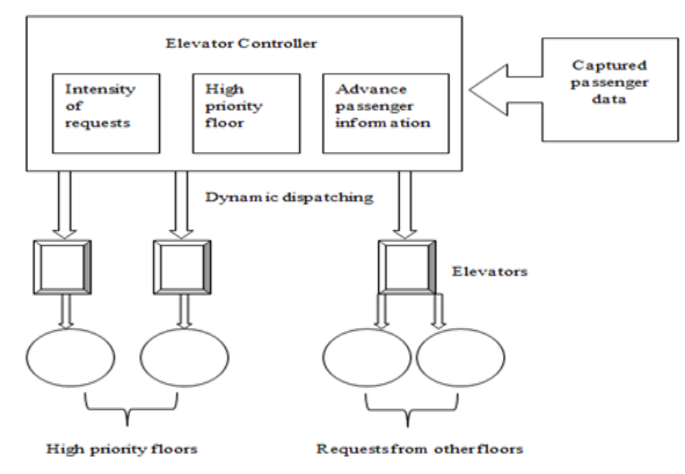

Figure 1. The architectural diagram of EGCS

The system captures the traffic data and identifies high priority floors. Depending on the intensity of requests, the EGCS allocates elevators dynamically to high priority floors. Figure 2 shows the dispatching model of elevator dispatching problem.

\section{RESEARCH METHOD}

The below model describes a high-level view of how the proposed elevator system framework works. For the elevator system, we consider, at a given point most of the traffic instigates from a single floor, 
and each elevator attends every floor. Thus, we can model the situation of high priority floor as many requests in the queue by a single floor, served by one or multiple identical elevators depending upon the intensity of requests. Consider a multi-elevator system each with the identical capacity of the passenger, functioning on $\mathrm{N}$ number of floors with value one as ground floor and $\mathrm{N}$ as the top floor. The functioning of the elevator dispatching system can be represented as;

N(f): Number of floors, fi: Building Floor, N(e): Number of elevators, E: Elevator

C: Number of request calls, Cf: Number of calls per floor, K: Number of elevators to allocate per

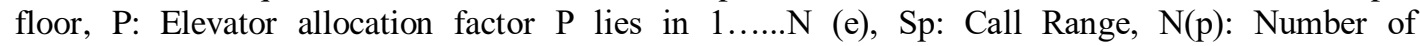
passengers,

$\lambda(p)$ : Intensity of passenger arrival,m(w): Maximum weight an elevator can carry,

$\mathrm{S}(\mathrm{f})$ : Source floor of travel, $\mathrm{T}(\mathrm{t})$ : Time of travel for an elevator from a source floor,

$\mathrm{C}(\mathrm{r})$ : Car call requests that arrive at a floor,

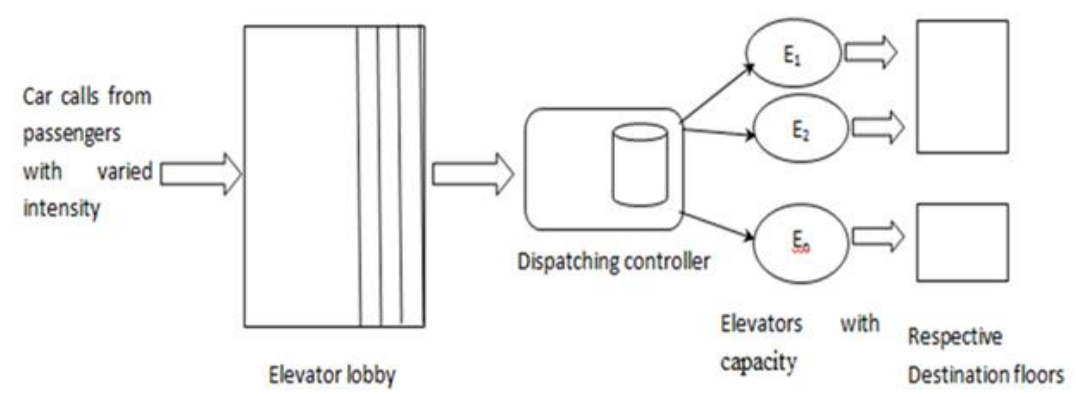

Figure 2. Model of elevator dispatching problem

The thorough algorithm for traffic-based elevator scheduling is explained below;

a) Initialize all the generated traffic information.

b) Evaluate observed traffic data and recognize high priority floors based on the intensity of requests for a particular floor. Count number of calls per floor $C_{f}$

c) Calculate the total number of calls in building $\mathrm{C}$

$$
\mathrm{C}=\sum_{f=0}^{N} C f
$$

Find call range for $\mathrm{P}=1 \ldots \ldots \mathrm{N}$ (e)

$\mathrm{S}_{\mathrm{p}}=(C \div N(e)) \times P$

Lift allocation for each floor $\mathrm{f}_{\mathrm{i}}, \mathrm{i}=1 \ldots N(f)$

For $\mathrm{P}=1 \ldots \ldots \mathrm{N}(\mathrm{e})$

If $\mathrm{S}_{\mathrm{p}-1}<\mathrm{C}_{\mathrm{f}}<\mathrm{S}_{\mathrm{p}}$ Then

$\mathrm{K}=\mathrm{N}(\mathrm{e})-(\mathrm{N}(\mathrm{e})-\mathrm{P})$ Where $\mathrm{S}_{0}=0$;

Assign $E_{0}, E_{1} \ldots \ldots E_{k}$ to respective floor $f_{i}$

d) If the call is coming from $\mathrm{f}_{\mathrm{i}}$, then check for the availability of elevators and assign elevator to identified priority floors. Move elevator to floor $f_{i}$ from the assigned elevator list based on the assignment algorithms.

e) Repeat the process whenever new data is obtainable.

The state-space $\mathrm{X}$ of the model for elevator assignment is obtained by outlining;

$C(r)$ The number of car call requests arriving at a floor,

$N(e)$ The number of elevators available to serve at that floor.

$X=\{(C(r), N(e))\}$

Consider a building of 20 floors with four working elevators.

Thus, $C(r)=0,1,2 \ldots . \quad N(e)=0,1,2,3,4$

When any number of requests comes at a floor $\mathrm{F}$, then the possible actions could be; 
Case 1: If a single car call request comes

Action $N(e)=0$ : Assign an elevator for request floor

Action $N(e)=1$ : This available elevator will serve the call

Action $N(e)=2$ : No action needed; an elevator will be on hold until the next request comes.

Case 2: If multiple requests come

When the intensity of the request is less than the maximum capacity of an elevator i.e.

$\lambda(p)<m(w)$ Then,

Elevator 1 will serve all the car call requests.

Thus, Elevator dispatches: $\{C(r), m(w)\}$

When the intensity of the request is greater than the maximum capacity of an elevator i.e.

$\lambda(p)>m(w)$ Then, Elevator 1 and 2 will serve the car call requests.

Thus, elevator dispatches: $\{C(r), 2(m(w))\}$

Case 3: Overflow situation: If the $\lambda(p)$ is more than the $m(w)$ of all the available elevators (assuming currently 2 elevators are available) i.e; $\lambda(p)>2(m(w))$

Elevator dispatches: $\{\mathrm{C}(\mathrm{r}), 2(\mathrm{~m}(\mathrm{w}))\}$

Leaving behind $\{C(r),(\lambda(p)-2(m(w)))\}$

\section{RESULTS AND DISCUSSIONS}

Consider a commercial building with 20 floors and 4 elevators serving the floors. There are a hospital, shopping mall and an office on floor number 10, 15 and 17 respectively. In a conventional elevator control system, each elevator serves all the floors based on the requests coming. Due to continuously varying circumstances, traffic flow diverges, thus working with such scheduling algorithms is less effective and results into long waiting time for passengers. As per the current building scenario, there will be high up peak traffic flow for floor number 15 during the morning, as everyone will be coming up at that time. Likewise, down peak traffic flow will be high in the evening for this floor when the official time overs. For the floor number 10, there will be variations in the call; however, as compared to other floors in the building, the up peak and down peak, the flow of traffic will be high in here. Typical considerations in the simulation are Number of Floors: 20, Number of Elevators: 4, The capacity of an Elevator: $540 \mathrm{~kg}$ (approx. 9 people), High traffic floors: 10 and 15. Figure 3 gives the pictorial view of the building with elevators.

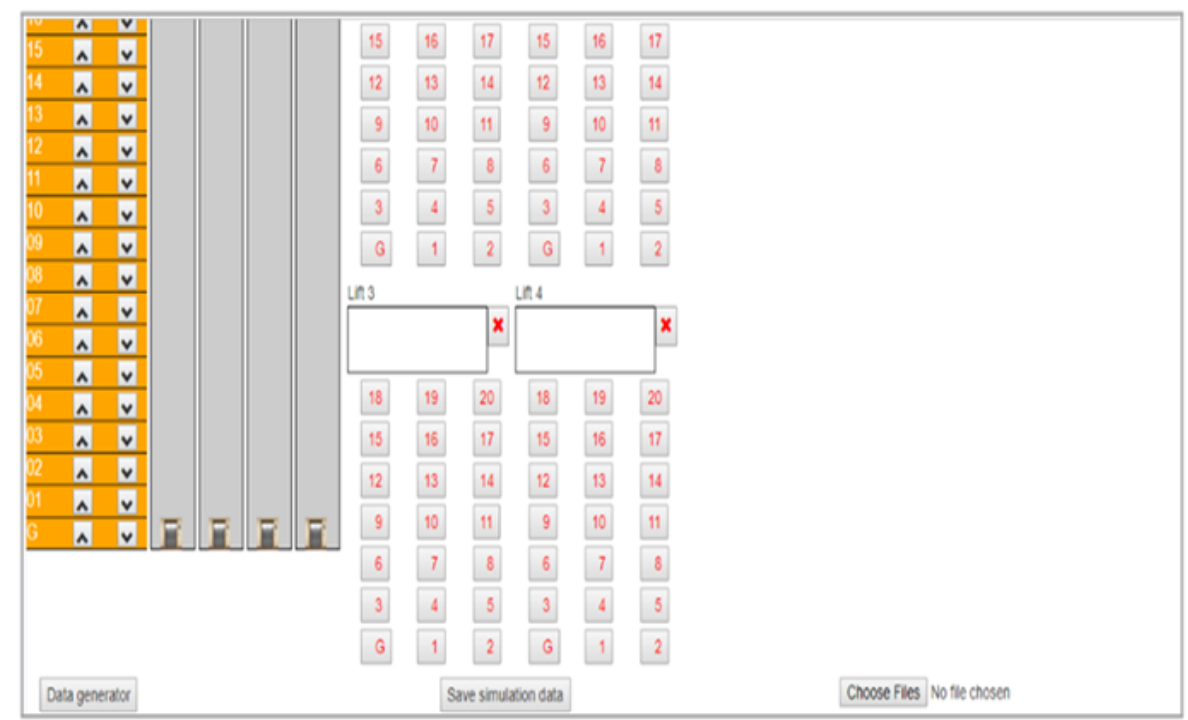

Figure 2. The pictorial view of the considered building

Here, for the testing purpose data is generated using the simulator, which generates random calls for all floors. Afterward, this generated data is saved in a file using the 'Save simulation Data' option shown below. An elevator controller uses this data to identify traffic patterns. Following is the snippet from the file showing how car call request are coming for each floor. With the same internals, the controller analyzes this data to get the advance traffic pattern. Figure 4 shows a sample of incoming calls. 
As per the graph shown in Figure 5, most of the calls are coming from floor number 10, 15 and 17 as compared to other floors. Thus, after analyzing this data, the controller will identify the priority floors as 10,15 and 17. So as per the intensity of coming request one explicit elevator will get allocated to each of these floors while the fourth elevator will then serve the requests coming from other floors. Here total car call requests coming are near about 1000 for a day. Moreover, as seen, out of this the request for floor number 10 , 15 and 17 collectively are 656 . So, on an average in a day $70 \%$ of requests come from these floors. Thus, with the proposed system, by an explicit allocation of an elevator to these floors $70 \%$ of requests will be served with minimum waiting time for passengers. This allocation shows a significant reduction in waiting time. Over the time, if the intensity of request increases for some other floors or even if the intensity reduces to these floors, then the controller identifies the priority floors as per the revised car call request data.

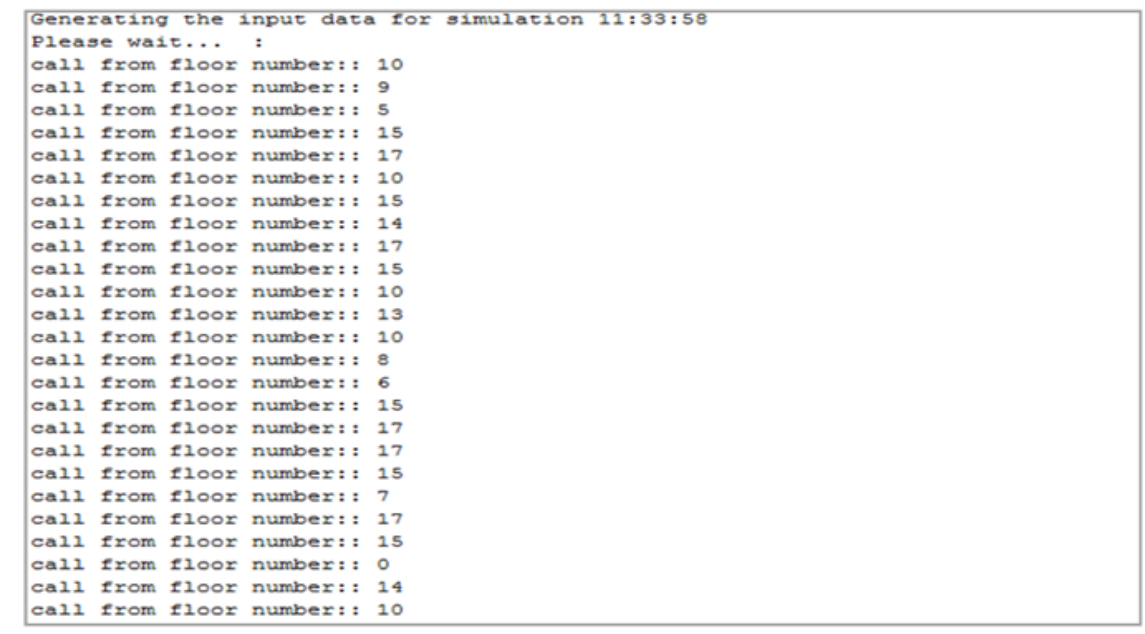

Figure 3. A sample of coming car call requests

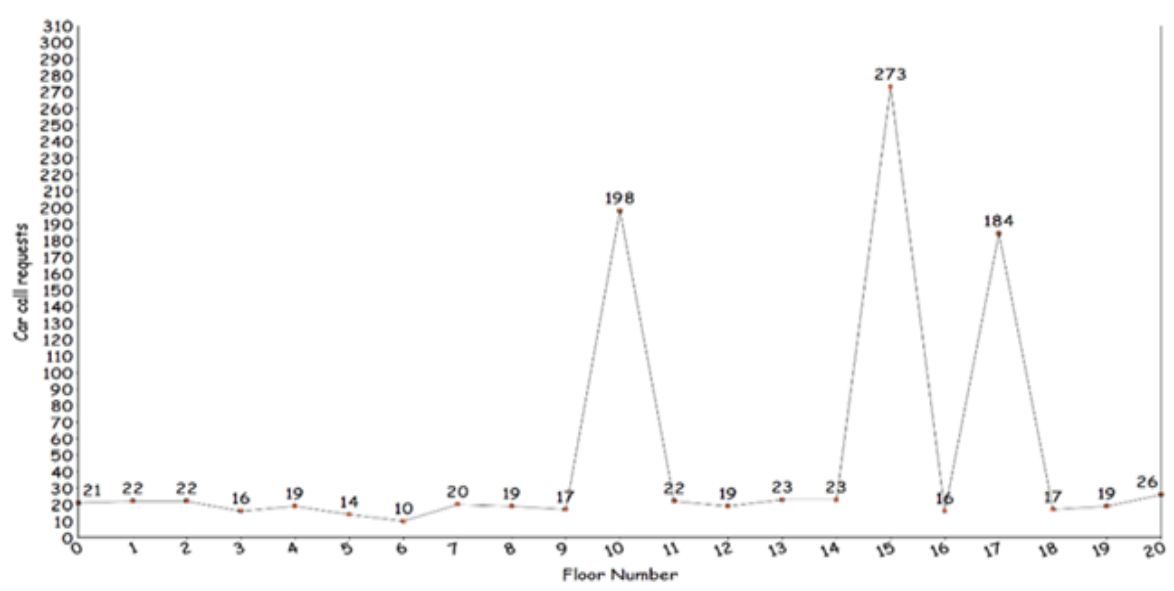

Figure 5. Observed traffic patterns

Thus, the presented approach will work for any general-purpose building. Table 1 shows the Elevator assignment based on observed traffic data. In even odd scheduling, even elevators are allocated to even floor calls, and odd Elevators are allocated to odd floor calls. The proposed system observed traffic pattern and based on traffic patterns elevator allocation is done. In Table 1 floor, no 15 have highest calls, so all four elevators are allocated to this floor. Floor no 10 and 17 are higher calls, so three elevators are allocated to these floors. Elevator number 2, 3, four are explicitly allocated to floor 10, 15 and 17. Remaining floors have minimum calls; hence, only one elevator is allocated to serve these calls. As floor no 10, 15 and 17 have maximum calls and explicitly allocated elevators, it minimizes the number of stops and results in minimum waiting time to serve these calls. 
Table 1. Elevator Allocation Based on Observed Traffic Data

\begin{tabular}{ccccc}
\hline Sr. No & Floor No & No. of calls & $\begin{array}{c}\text { Allocated Elevator E } \\
\text { (Even/Odd scheduling) }\end{array}$ & $\begin{array}{c}\text { Allocated Elevator E } \\
\text { (traffic-based scheduling) }\end{array}$ \\
\hline 1 & 1 & 23 & E 1, E 3 & E 1 \\
2 & 2 & 16 & E 2, E 4 & E 1 \\
3 & 3 & 12 & E 1, E 3 & E 1 \\
4 & 4 & 15 & E 2, E 4 & E 1 \\
5 & 5 & 16 & E 1, E 3 & E 1 \\
6 & 6 & 20 & E 2, E 4 & E 1 1 \\
7 & 7 & 10 & E 1, E 3 & E 1 \\
8 & 8 & 29 & E 2, E 4 & E 1 \\
9 & 9 & 23 & E 1, E 3 & E 1 $~$ \\
10 & 10 & 200 & E 2, E 4 & E 1 \\
11 & 11 & 18 & E 1, E 3 & E 1 \\
12 & 12 & 17 & E 2, E 4 & E 1 \\
13 & 13 & 22 & E 1, E 3 & E 1 , E 4 \\
14 & 14 & 24 & E 2, E 4 & E 2, E 3, E 4 \\
15 & 15 & 269 & E 1, E 3 & E 1 \\
16 & 16 & 24 & E 2, E 4 & E 1 \\
17 & 17 & 187 & E 1, E 3 & E 1 \\
18 & 18 & 17 & E 2, E 4 & E 1, E 3 \\
19 & 19 & 16 & E 2, E 4 & \\
20 & 20 & 25 & &
\end{tabular}

Consider calls are coming from each floor and elevator allocation is as per Table 1. Table 2 shows the comparative study of waiting time required to serve the calls at floor 10, 15 and 17 using even-odd elevator scheduling and traffic-based elevator scheduling. As compared to even odd elevator scheduling, the traffic-based elevator scheduling reduces the waiting time of passengers. Waiting time analysis between even/odd and traffic-based scheduling is shown in Figure 6.

Table 2. Waiting Time (WT) in Even Odd and Traffic-Based Scheduling

\begin{tabular}{cccc}
\hline Sr. No & Calling Floor & WT (Even/Odd scheduling in a sec) & WT (traffic-based scheduling in a sec) \\
\hline 1 & $0 \rightarrow 10$ & 28 & 20 \\
2 & $0 \rightarrow 15$ & 44 & 30 \\
3 & $0 \rightarrow 17$ & 50 & 34 \\
4 & $10 \rightarrow 0$ & 28 & 20 \\
5 & $15 \rightarrow 0$ & 44 & 30 \\
6 & $17 \rightarrow 0$ & 50 & 34 \\
7 & AVG WT & 40.66 & 28 \\
\hline
\end{tabular}

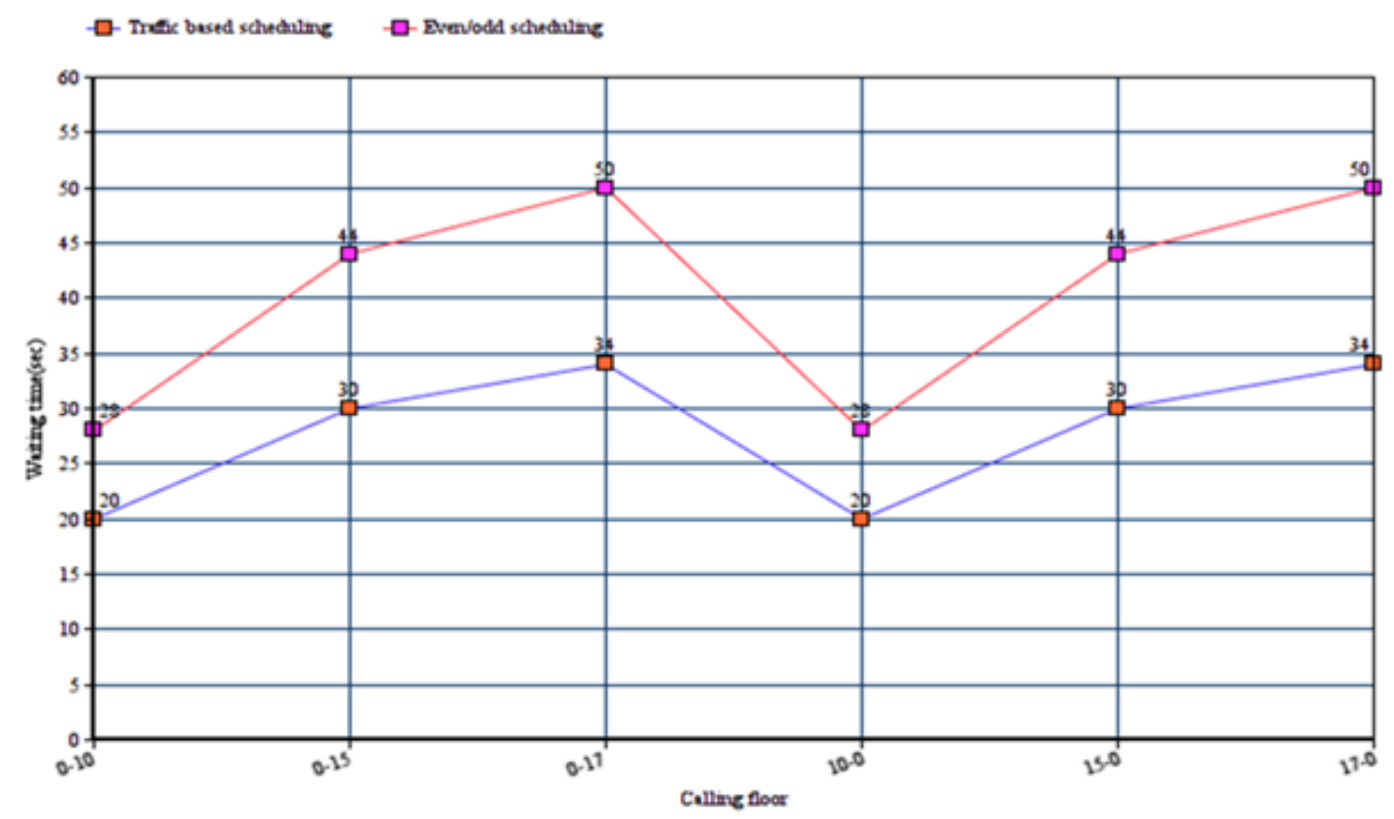

Figure 6. Waiting time analysis between even/odd and traffic-based scheduling 


\section{CONCLUSION}

We have presented a novel approach to optimize the dispatching of cars in an Elevator Group Control System. This approach serves the high-rise buildings with differential traffic at each level and in peak traffic hours. Implementation considers a hall call pattern to define the best possible dispatching strategy. The approach is successfully simulated for 20-floor building, and it outperforms the existing computing techniques. The algorithm reduces the cumulative wait time for passengers by using traffic based floor preference approach.

\section{REFERENCES}

[1] Barney, G. Elevator Traffic Handbook, Abingdon: Taylor \& Francis, 2003.

[2] Liting Cao, Shiru Zhou, Shuo Yang, "Elevator Group Dynamic Dispatching System Based on Artificial Intelligent Theory," 2008 International Conference on Intelligent Computation Technology and Automation (ICICTA), 2008, 10.1109/ICICTA.2008.114.

[3] Emre Oner Tartan, Cebrail Ciftlikli, “A Genetic Algorithm Based Elevator Dispatching Method For Waiting Time Optimization," International Federation of Automatic Control, Hosting by Elsevier Ltd., vol. 49, no. 3, pp. $424-$ 429, 2016, [Online], Available DOI: 10.1016/j.ifacol.2016.07.071.

[4] Jinghong Zheng. Ho Chee Tat Thomas, Yuan HuaiBing, "Traffic Prediction for Efficient Elevator Dispatching," Proceedings of TENCON 2018 - IEEE Region 10 Conference Jeju, Korea, 28-31 Oct 2018.

[5] Yerzhigit Bapin, Vasilios Zarikas, "Smart Building's Elevator with Intelligent Control Algorithm based on Bayesian Networks," International Journal of Advanced Computer Science and Applications, vol. 10, no. 2, 2019

[6] Zhenshan Yang, Wenjiao Yue, "Elevator Traffic pattern recognition based on Fuzzy BP Neural Network with SOM Algorithm," AMSE JOURNALS-AMSE IIETA publication-2017-Series: Advances B, vol. 60, no 4, pp 630-645, 2017.

[7] Toni Tukia, Semen Uimonen, Marja-Liisa Siikonen, Claudio Donghi, and Matti Lehtonen, "High-resolution modeling of elevator power consumption," Journal of Building Engineering, 2018, [Online], Available DOI: 10.1016/j.jobe.2018.03.008.

[8] Mirko Ruokokoski, Janne Sorsa, Marja-Liisa Siikonen, Harri Ehtamo, "Assignment Formulation for the Elevator Dispatching Problem with Destination Control and Its Performance Analysis," European Journal of Operational Research, 2016, [Online], Available DOI: 10.1016/j.ejor.2016.01.019.

[9] M. D. Sale, V. chandra Prakash "Elevator Group Control System (EGCS): scheduling of elevators using even and odd elevators approach," Journal of Advanced research in Dynamical and Control Systems (JARDCS 2017), no. 18, pp. 3231-3242, 2017.

[10] M. D. Sale, V. Chandra Prakash, "Dynamic Scheduling of Elevators with Reduced Waiting Time of Passengers in Elevator Group Control System: Fuzzy System Approach," Innovations in Computer Science and Engineering: Proceedings of the Fourth ICICSE 2016 Volume 8 of Lecture Notes in Networks and Systems, Springer, pp. 339-346, 2017.

[11] Arnaud Malapert, Juha-Matti Kuusinen, "Estimation of elevator passenger traffic based on the most likely elevator trip origin-destination matrices," Journal of Building Services Engineering Research \& Technology, Building Services Engg. Research Technology, sage publications, 2017, [Online], Available DOI: $10.1177 / 0143624417707875$.

[12] Sungyong Ahn, Soyoon Lee and Hyokyung Bahn, "A smart elevator scheduler that considers dynamic changes of energy cost and user traffic," Integrated Computer-Aided Engineering, vol. 24, no. 2, pp. 187-202, 2017, [Online], Available DOI 10.3233/ICA-170539.

[13] Jinglong Zhang and Qun Zong, "Energy-saving-oriented group-elevator dispatching strategy for multi-traffic patterns," Journal of Building Services Engineering Research \& Technology, 2014, [Online], Available DOI: $10.1177 / 0143624414526723$.

[14] Choongwan Koo, Taehoon Hong, Jaeseong Yoon, "Zoning-Based Vertical Transportation Optimization for Workers at Peak Time in a Skyscraper Construction," Computer-Aided Civil and Infrastructure Engineering, 2016, [Online], Available DOI: 10.1111/mice.12220.

[15] Albert So, Lutfi Al-Sharif, Ahmad Hammoudeh, "Traffic analysis of a three-dimensional elevator system," Journal of Building Services Engineering Research \& Technology, 2017, [Online], Available DOI: $10.1177 / 0143624417710106$.

[16] Hailiang Dong at.al.," Towards the Design of Optimal Range Assignment for Elevator Groups under Fluctuant Traffic Loads," 23rd International IEEE Conference on Embedded and Real-Time Computing Systems and Applications Taiwan, 2017

[17] Lutfi Al-Sharif, "A Three Element New Paradigm for Evaluating the Performance of Up-Peak Elevator Group Control Algorithms," Lift Report 2016, vol. 42(4), pp.50-58, 2016. 